\title{
Behavioural Analysis of the Modified Lotka-Volterra Predator-Prey Model Using a Computer Simulation
}

JOURNAL OF RESEARCH

\section{Olabanjo Olusola A. ${ }^{1}$, Wusu Ashiribo S. ${ }^{2}$, Akanbi Moses A. ${ }^{2}$ and Aribisala Benjamin S. ${ }^{1}$}

${ }^{1}$ Computer Science Department, Lagos State University, Ojo. Lagos, Nigeria.

${ }^{2}$ Department of Mathematics, Lagos State University, Ojo. Lagos, Nigeria.

\section{Correspondence}

Olabanjo Olusola A., Department of Computer Science, Faculty of Science, Lagos State University, Nigeria.

Email:solarmic2015@gmail.com

\section{Funding information}

This research work is self sponsored.

\section{Abstract:}

Introduction: In a real-world scenario, problems are identified, assumptions are made, data are collected and models are proposed. The underlying mathematical structure of the models is analyzed to appraise the sensitivity of the conclusions when the assumptions are not precisely met.

Aims: In this work, we present the formulation of a modified Lotka-Volterra Predator-Prey Model to include terms which represent the degrees of internal competition of both predators and prey for their limited resources and a simulation of the stability behaviour of the resulting system.

Materials and Methods: A computer simulation of the behaviour - stability of the solution of the model - of the system under different conditions is also presented. The critical points, eigenvalues and phase portrait of the system in terms of the model parameters are also obtained.

Results: The model interpretation and applicability are also discussed.

Conclusion: The trajectories of the proposed model are not periodic and tend to equilibrium level with time, thus, making the system to be asymptotically stable. This behaviour is in line with the observable natural predator-prey system.

Keywords: Lotka-Volterra, Predator-Prey Model, Simulation, Critical Points, Eigenvalues, Stability

All co-authors agreed to have their names listed as authors.

This is an open access article under the terms of the Creative Commons Attribution License, which permits use, distribution and reproduction in any medium, provided the original work is properly cited.

(c) 2018 The Authors. Journal of Research and Reviews in Science - JRRS, A Publication of Lagos State University 


\section{INTRODUCTION}

Interest in how populations tend to grow gained attention in the 18th century when the work of the author in [1] was published. The author in his work [1], put forth an exponential growth model for human population and concluded that eventually the population would exceed the capacity to grow an adequate food supply.

However, the assumptions of the model left out factors (environment and interaction between population of different species that are important to population growth and hence it could not stand the test of time as it was proven to be inaccurate for technologically developed countries.

Interestingly, the work [1] stimulated interest in the study of the ecological problem of a predator population interacting with the prey one which has been of great interest in many areas of science. Early attempt to study this problem using mathematical equations is attributed to the independent works of [2] and [3, 4]. The authors independently produced the equations that gave the model of this problem and discovered that, under simple hypotheses, periodic fluctuations of the populations occur. Hence, the mathematical equations which model the struggle for existence of two species (predator and prey) bear the names of the pioneer scientists: Lotka and Volterra. The factors that were left out in the model proposed by the author in [1] formed the basis for the formulation of the Lotka-Volterra model. The assumptions about the environment and evolution of the predator and prey population for the Lotka-Volterra model are:

- The prey population have an unlimited food supply at all times.

- The prey population $x(t)$ increases at a rate $d x=a x(t) d t$ (proportional to the number of prey) but is simultaneously destroyed by predators at a rate $d x=-b x(t) y(t) d t$ (proportional to the product of the numbers of predator and prey).

- The predator population $y(t)$ decreases at a rate $d y=$ $-c y(t) d t$ (proportional to the number of predators), but increases at a rate $d y=d x(t) y(t) d t$ (again proportional to the product of the numbers of predator and prey).

The first and simplest predator-prey model ([2], [3], [5], [6]) is given by the nonlinear system of first-order ordinary differential equations

$$
\begin{aligned}
& \frac{d x}{d t}=a x(t)-b x(t) y(t) \\
& \frac{d y}{d t}=-c y(t)+d x(t) y(t),
\end{aligned}
$$

In this work, we present a simulation of the stability behaviour of a modified version of the predator-prey model.

\section{MATERIAL AND METHODS}

\subsection{Identification of Problem}

The author in [7] noted that as more individuals are produced than can possibly survive, there must in every case be a struggle for existence. This is true as the individuals are faced with the need to compete for their limited resources either with species of same kind or distinct species. In this work, we take a closer look at the situation between the predator and prey. The predators eat the prey and the prey live on the abundantly available natural food in the environment. If the predators eat so many of the prey that the prey cease to be abundant, the food supply of the predators is greatly reduced, then the predator will starve. A dwindling in the population of the predator will result in a significant increase in the prey's population. As the prey population increases, the food supply of the predator grows and so does the predators' population. Also, more predators are eating increasingly more prey again. In this cyclic situation, one asks whether the cycle continues indefinitely or does one of the species eventually go into extinction. The ability to answer the above question finds relevance in many areas of science.

\subsection{Model Formulation and Assumptions}

It was observed that internal competitions between predator and prey for their limited resources were not considered as major factors in the models earlier proposed. In this work, we considered a modified Lotka-Volterra equation by introducing the degree of internal competition of the predator and prey for their limited resources. We assume that the degree of internal competition of the predator and prey are proportional to the square of the predator and prey populations, i.e. $f y^{2}(t)$ and $e x^{2}(t)$ respectively. Hence, the modified Lotka-Volterra predator-prey model presented in this work is given as

$$
\begin{aligned}
& \frac{d x}{d t}=a x(t)-b x(t) y(t)-e x^{2}(t) \\
& \frac{d y}{d t}=-c y(t)+d x(t) y(t)-f y^{2}(t),
\end{aligned}
$$

where $a, b, c, d, e, f$ are positive constants.

\subsection{Stability Property of Model Solution}

To analyse the behaviour of the system under consideration, some properties of the system for varying parameters of the model need to be investigated. Here, we present the critical points, eigenvalues, phase portrait and stability of the model under consideration. 


\subsubsection{Critical Points of Model}

To obtain the critical points of the model (2), we set $d x / d t=$ $f(x, y)=0$ and $d y / d t=g(x, y)=0$ and solve. Unlike a linear system, a nonlinear system could have none, one, two, three, or any number of critical points. Since there might be multiple critical points present on the phase portrait, each trajectory could be influenced by more than one critical point. This results in a much more chaotic appearance of the phase portrait. Consequently, the type and stability of each critical point need to be determined locally on a case-by- case basis. Considering the system

$$
\begin{aligned}
& \frac{d x}{d t}=a x(t)-b x(t) y(t)-e x^{2}(t)=0 \\
& \frac{d y}{d t}=-c y(t)+d x(t) y(t)-f y^{2}(t)=0,
\end{aligned}
$$

Since the solutions $x(t)$ and $y(t)$ are not easily obtained by mere inspection, we proceed with the use of Mathematica ${ }^{R}$ 's built-in command for solving equations. Solving the system above for $x(t)$ and $y(t)$ results in four critical points of the system. Hence, the critical point of the model (2) are

$$
\begin{aligned}
& \left(x_{1}, y_{1}\right)=\left(0,-\frac{c}{f}\right) \\
& \left(x_{2}, y_{2}\right)=\left(-\frac{-a f-b c}{b d+e f},-\frac{c e-a d}{b d+e f}\right) \\
& \left(x_{3}, y_{3}\right)=(0,0) \\
& \left(x_{4}, y_{4}\right)=\left(\frac{a}{e}, 0\right)
\end{aligned}
$$

Interestingly, three of the critical points of the system are dependent on the values of the parameters $a, b, c, d, e, f$.

\subsubsection{Eigenvalues of Model}

The matrix

$$
J(x, y)=A\left(\begin{array}{cc}
\partial_{x} f(x, y) & \partial_{y} f(x, y) \\
\partial_{x} g(x, y) & \partial_{y} g(x, y)
\end{array}\right)
$$

is called the Jacobian matrix of the system. It contains the first order partial derivatives of $f$ and $g$ evaluated at the point $(x, y)$. Now

$$
J(x, y)=\left(\begin{array}{cc}
a-b y-2 e x & -b x \\
d y & -c+d x-2 f y
\end{array}\right)
$$

Evaluating (6) respectively at the four critical points (4) results in

$$
\begin{gathered}
J_{1}(x, y)=\left(\begin{array}{cc}
a+\frac{b c}{f} & 0 \\
-\frac{c d}{f} & c
\end{array}\right), \\
J_{2}(x, y)=\left(\begin{array}{cc}
-\frac{e(a f+b c)}{b d+e f} & -\frac{b(a f+b c)}{b d+e f} \\
\frac{d(a d-c e)}{b d+e f} & \frac{c e f-a d f}{b d+e f}
\end{array}\right)
\end{gathered}
$$

$$
\begin{gathered}
J_{3}(x, y)=\left(\begin{array}{cc}
a & 0 \\
0 & -c
\end{array}\right) \\
J_{4}(x, y)=\left(\begin{array}{cc}
-a & -\frac{a b}{e} \\
0 & \frac{a d}{e}-c
\end{array}\right)
\end{gathered}
$$

The eigenvalues of (7), (8), (9) and (10) respectively are

$$
\begin{aligned}
\lambda_{1} & =c \\
\mu_{1} & =\frac{a f+b c}{f}
\end{aligned}
$$

$$
\begin{gathered}
\lambda_{2}=\frac{1}{2(b d+e f)}(-b c e-a d f-a e f+c e f- \\
\sqrt{ }\left((a d f+a e f+b c e-c e f)^{2}-\right. \\
\left.\left.4\left(a^{2} b d^{2} f+a^{2} d e f^{2}+a b^{2} c d^{2}-a c e^{2} f^{2}-b^{2} c^{2} d e-b c^{2} e^{2} f\right)\right)\right) \\
\mu_{2}=\frac{1}{2(b d+e f)}(-b c e-a d f-a e f+c e f+ \\
\sqrt{ }\left((a d f+a e f+b c e-c e f)^{2}-\right. \\
\left.\left.4\left(a^{2} b d^{2} f+a^{2} d e f^{2}+a b^{2} c d^{2}-a c e^{2} f^{2}-b^{2} c^{2} d e-b c^{2} e^{2} f\right)\right)\right) \\
\lambda_{3}=a \\
\mu_{3}=-c \\
\lambda_{4}=-a \\
\mu_{4}=\frac{a d-c e}{e}
\end{gathered}
$$

\subsection{Stability of Model}

We can use the eigenvalues of the Jacobian matrix to decide the type and stability of the critical point $(x, y)$. Let $\lambda$ and $\mu$ be the two eigenvalues. Assume that $\lambda$ and $\mu$ are different from 0 .

\subsubsection{Phase Portrait and Field Directions of the Model}

The best way to describe the vector field of the system is to simulate the plot with varying values of the parameter. Since a static plot of the vector field for the model can only be obtained by fixing the values of the parameter, we present some of the possible scenarios with three case: 
Table 1: Conditions on eigenvalues to ensure different stability

\begin{tabular}{|c|c|c|}
\hline Eigenvalues & Linear System & Nonlinear System \\
\hline \multicolumn{3}{|c|}{$\lambda, \mu \in \mathbb{R}$} \\
\hline$\lambda>\mu>0$ & Nodal Source (Unstable) & Nodal Source (Unstable) \\
\hline$\lambda<\mu<0$ & Nodal Sink (Stable) & Nodal Sink (Stable) \\
\hline$\lambda>0>\mu$ & Saddle point (Unstable) & Saddle point (Unstable) \\
\hline$\lambda=\mu>0$ & $\begin{array}{l}\text { Degenerate Source or Nodal } \\
\text { Source (Unstable) depending of } \\
\text { the geometric multiplicity of } \lambda\end{array}$ & $\begin{array}{l}\text { Source (Degenerate, Nodal, } \\
\text { Spiral Source depending on the } \\
\text { nonlinear terms) }\end{array}$ \\
\hline$\lambda=\mu<0$ & $\begin{array}{l}\text { Degenerate Sink or Nodal Sink } \\
\text { (Stable) depending of the geo- } \\
\text { metric multiplicity of } \lambda\end{array}$ & $\begin{array}{l}\text { Sink (Degenerate, Nodal, Spiral } \\
\text { Sink) (Stable depending on the } \\
\text { nonlinear terms) }\end{array}$ \\
\hline \multicolumn{3}{|c|}{$\lambda, \mu \in \mathbb{C}$} \\
\hline $\operatorname{Re}(\lambda)>0$ & Spiral Source (Unstable) & Spiral Source (Unstable) \\
\hline $\operatorname{Re}(\lambda)<0$ & Spiral Sink (Stable) & Spiral Sink (Stable) \\
\hline $\operatorname{Re}(\lambda)=0$ & Center (Stable) & $\begin{array}{l}\text { Center, Spiral Sink, Spiral } \\
\text { Source (Stability cannot be de- } \\
\text { termined based on } \lambda \text { ) }\end{array}$ \\
\hline
\end{tabular}

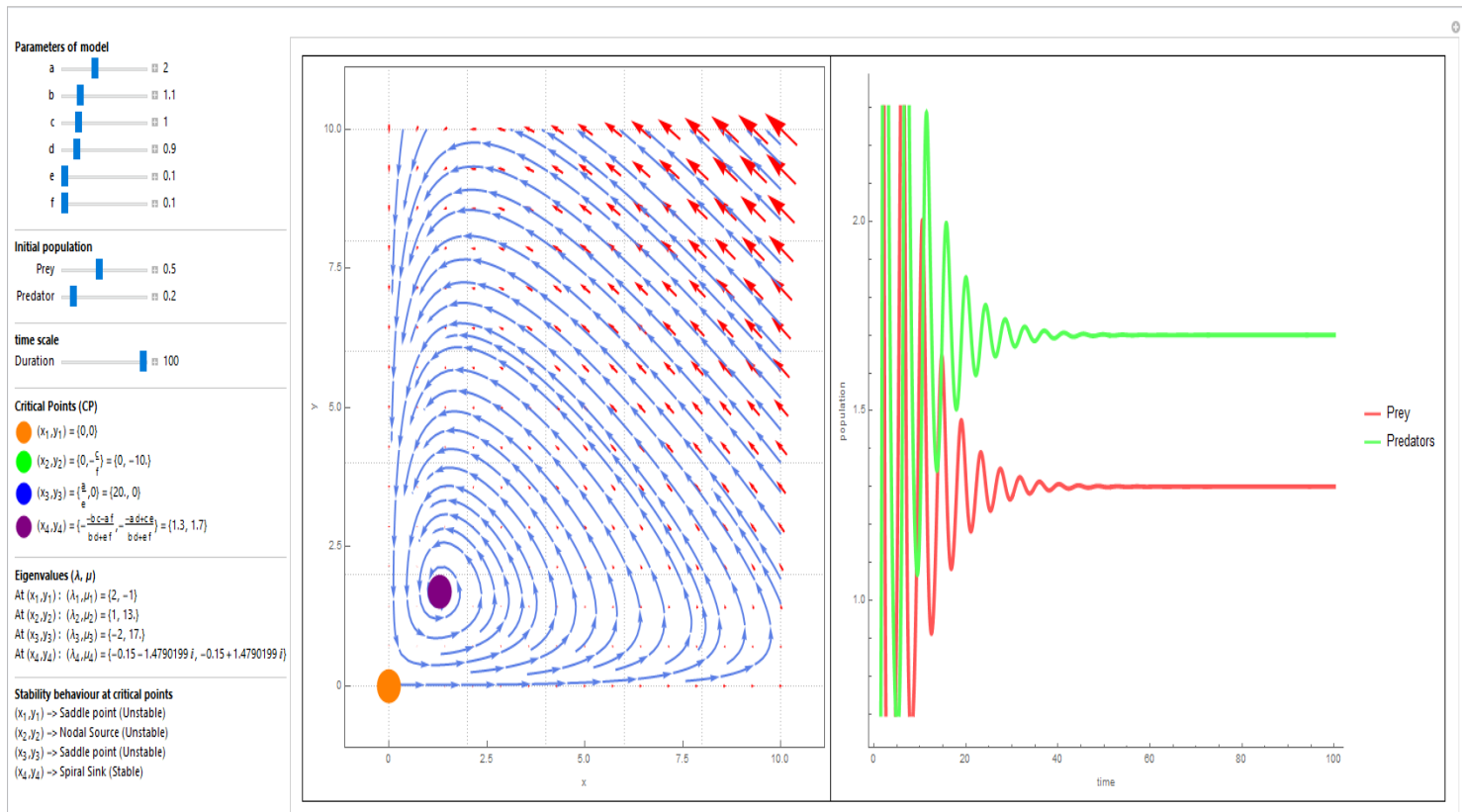

Figure 1: Phase portrait and population plots for (2) with $a=2, b=1.1, c=1, d=0.9, e=f=0.1$

\section{RESULTS}

\subsection{Model Interpretation and Conclusion}

Figures 1, 2 and 3 show that the stability at the various critical depends on the values of the model's parameters. Based on the introduction of the degree of internal competition between the predator and prey, we see from Figures 1, 2 and 3 , that the trajectories of the model are not periodic and tend to equilibrium level with time. Hence, the system (2) is asymptotically stable. This behaviour is in line with the observable natural predator-prey system.

\section{CONCLUSION}

We have been able to modify the classical Lotka-Volterra equation by introducing terms which represent degrees of internal competition between the predator and prey. Unlike the classical model, this modification has been shown to make the system asymptotically stable. This behaviour conforms to the observable natural predator-prey system. For future 


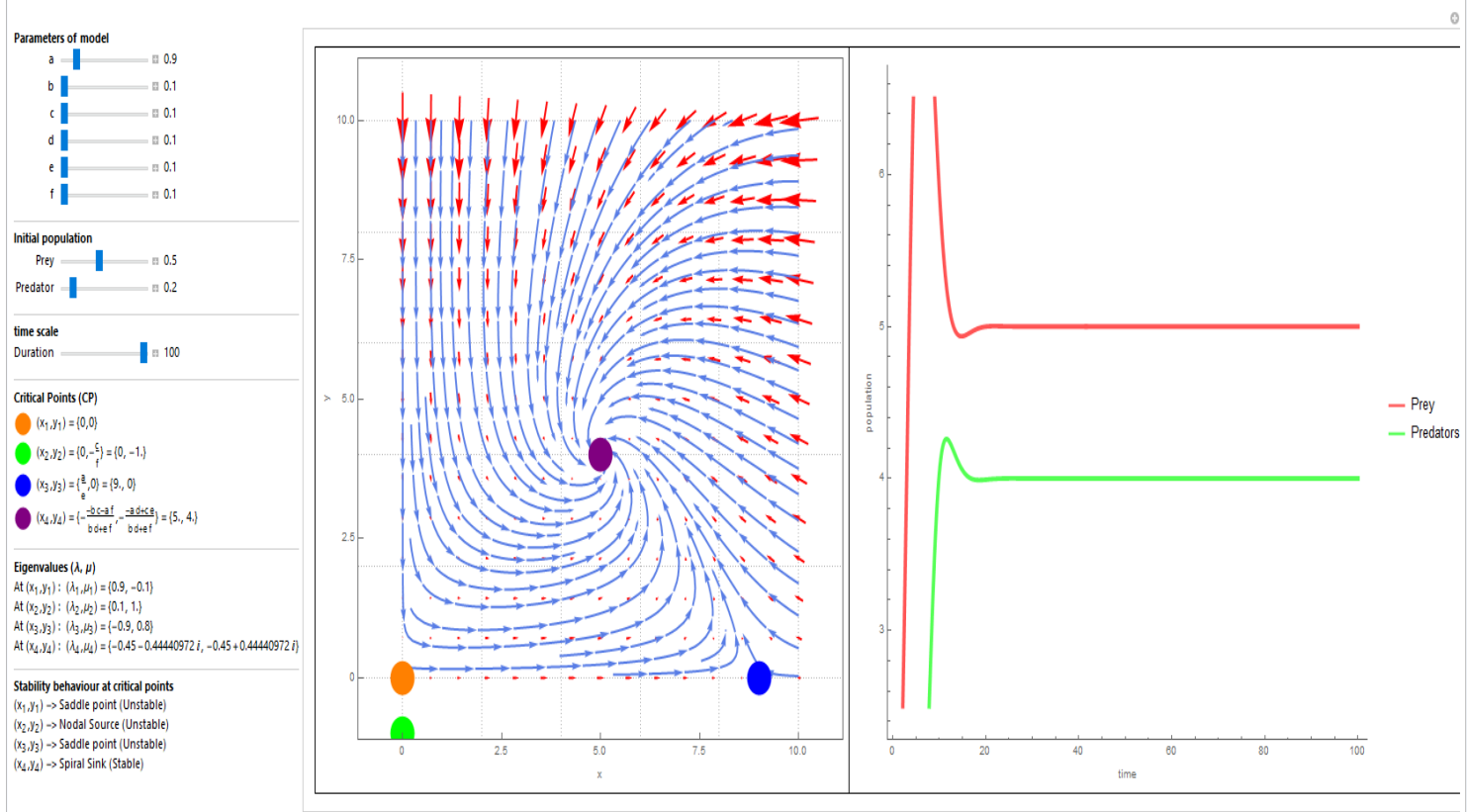

Figure 2: Phase portrait and population plots for (2) with $a=0.9, b=0.1, c=0.1, d=0.1, e=f=0.1$

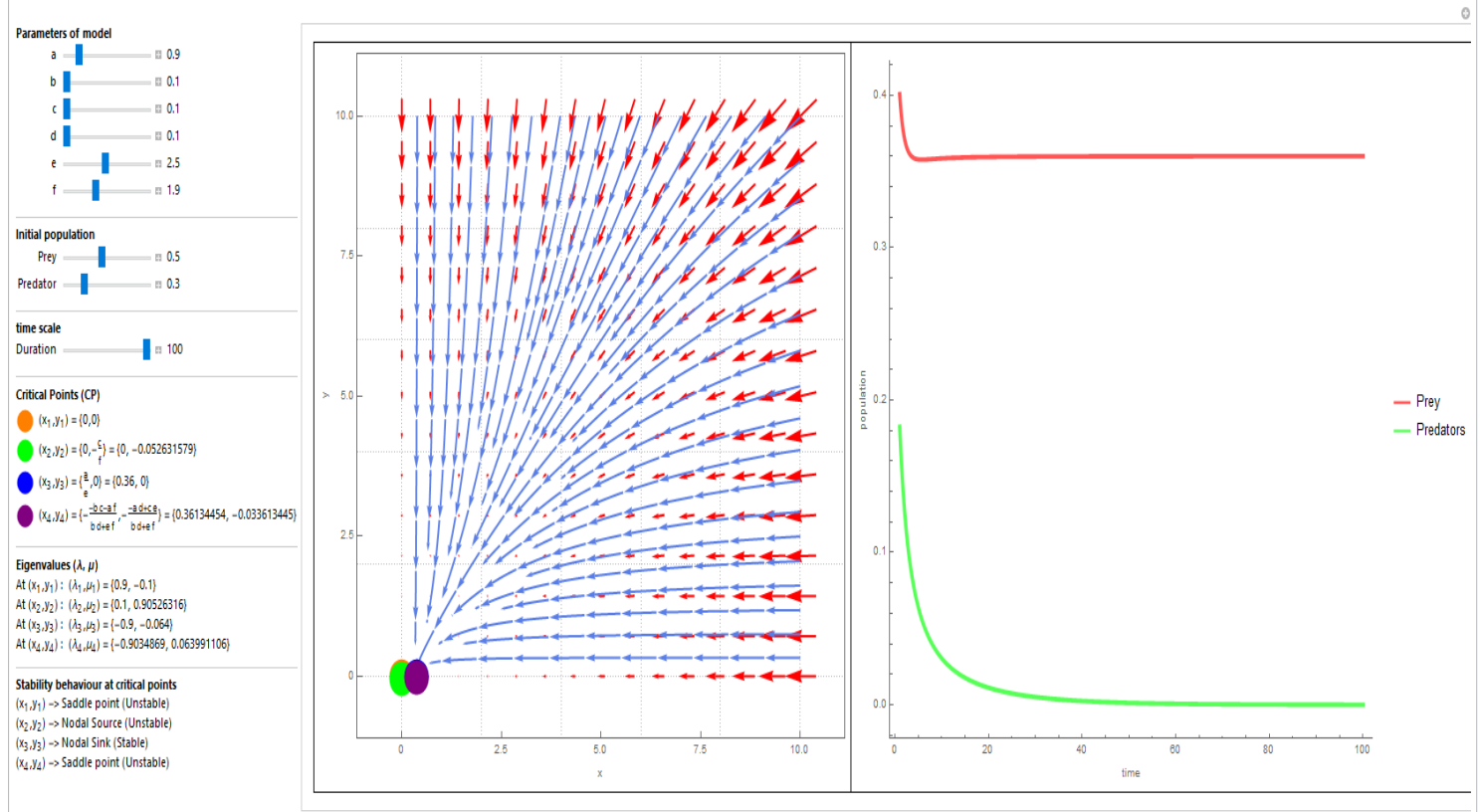

Figure 3: Phase portrait and population plots for (2) with $a=0.9, b=0.1, c=0.1, d=0.1, e=2.5, f=1.9$

work, more variables can be included in order to get a better representation of the system.

\section{AUTHORS' CONTRIBUTIONS}

All authors participated actively in this research work and the writing of the manuscript. 


\section{CONSENT (WHERE EVER APPLICABLE)}

Consent form has been approved by all authors.

\section{REFERENCES}

[1] Malthus T. R., An essay on the principle of population as it affects the future improvement of society, London, Printed for J. Johnson, in St. Paul's Church-yard, 1798.

[2] Lotka, A. J., Analytical note on certain rhythmic relations in organic systems, Proc. Nat. Acad. 1920; 6:410-415.

[3] Volterra, V., Fluctuations in the abundance of a species considered mathematically, Nature, 1926; 118:558560.

[4] Volterra, V., Sulle fluttuazioni biologiche, Rendiconti del Seminario Matematico e Fisico di Milano, 1929; 3(1):154-174.

[5] Kingsland, Sh. E., Modeling nature, University of Chicago Press, 1995.

[6] Boyce W, E., DiPrima R. C., Elementary differential equations and boundary value problems, John Wiley \& Sons, Inc., New York. 2001.

[7] Darwin, C., On the origin of species by means of natural selection, or, the preservation of favoured races in the struggle for life, London: J. Murray., 1859. 